\title{
See-Sedimente als Zeugen der Schadstoffbelastung
}

\author{
Christian Bogdal, Peter Schmid, Flavio S. Anselmetti, Martin Scheringer
}

Schadstoffmessungen in den Sedimenten von Seen ermöglichen es, die Eintragsgeschichte von

Chemikalien zu rekonstruieren. In Gletscherseen steigt die Konzentration persistenter organischer

Schadstoffe, die längst verboten sind.

Wenig wasserlösliche und langlebige Stoffe gelangen über Luft, Regen und Flusswasser in die Seen und lagern sich dort in den Sedimenten ab. Sedimentsuntersuchungen zeigen daher, wie stark ein Gewässer mit Chemikalien belastet ist. Ist bekannt, in welchen Konzentrationen die Chemikalien im Sediment vorliegen und aus welchen Jahren die Sedimente datieren, dann lässt sich rekonstruieren, wann welche Mengen dieser Substanzen in die Umwelt gelangt sind.

\section{Schwebstoffe: Biomasse oder Mineralien}

- Je nach Art des Gewässers ist die Zusammensetzung der sedimentierten Schwebstoffe unterschiedlich: In Gewässern mit starker biologischer Aktivität enthalten die Sedimente vorwiegend Biomasse und somit einen hohen Anteil an organischem Kohlenstoff. Ganz anders in proglazialen Seen, das sind Seen, die sich durch das Abschmelzen eines Gletschers bilden (Abbildung 1): Hier enthalten die Sedimente vorwiegend mineralische Schwebstoffe aus dem Gesteinsabrieb, der aus dem Gletscher über das Schmelzwasser eingespült wird.

Wichtige global vorkommende langlebige Chemikalien sind die persistenten organischen Schad-

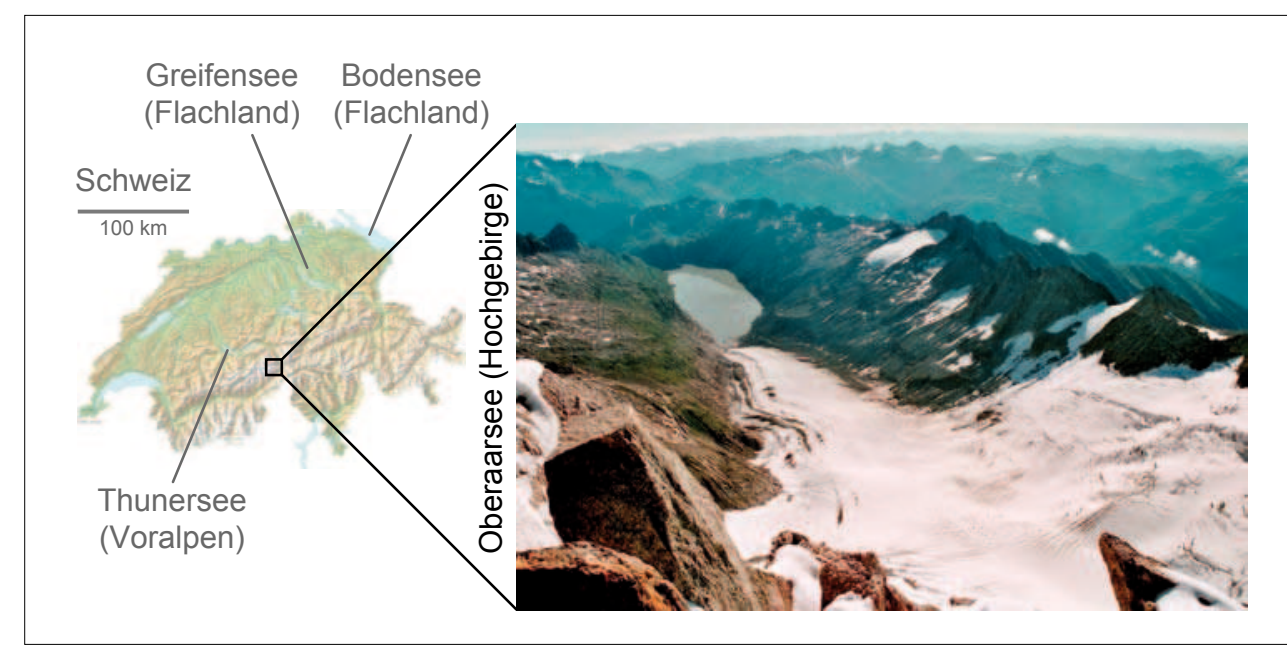

Abb. 1. Karte der Schweiz mit den untersuchten Seen (links); Oberaargletscher und Oberaarsee im Berner Oberland. (Foto: Sandro Hügli, Interlaken)

stoffe (persistent organic pollutants, POPs). $\mathrm{Zu}$ diesen zählen polychlorierte Biphenyle (PCB), das Insektizid Dichlordiphenyltrichlorethan (DDT) und die hochtoxischen polychlorierten Dibenzop-dioxine und Dibenzofurane (PCDD/F), die bei Verbrennungen entstehen.

Die POPs wurden bis in die 1970er Jahre in beträchtlichem Umfang eingesetzt und in die Umwelt freigesetzt. Seit der Stockholmer Konvention, die 2004 in Kraft trat, sind sie weltweit stark eingeschränkt. Wegen ihrer Persistenz sind diese Stoffe jedoch auch nach den Anwendungsverboten nicht aus der Umwelt verschwunden.

\section{Auf mehreren \\ Wegen in die Seen}

- POPs sind mittelflüchtige organische Verbindungen (semivolatile organic compounds, SVOCs). Daher verbreiten sich diese Stoffe weiträumig über atmosphärischen Transport und anschließende Ablagerung in Regen oder Schnee. Sie adsorbieren auch an Aerosolpartikel und breiten sich mit ihnen aus. Bei niedrigen Temperaturen ist der Dampfdruck der Substanzen niedriger, und Abbauprozesse laufen nur noch langsam ab - daher reichern sich SVOCs in den Polarregionen an.

In stehenden Gewässern adsorbieren POPs an Schwebstoffe und 


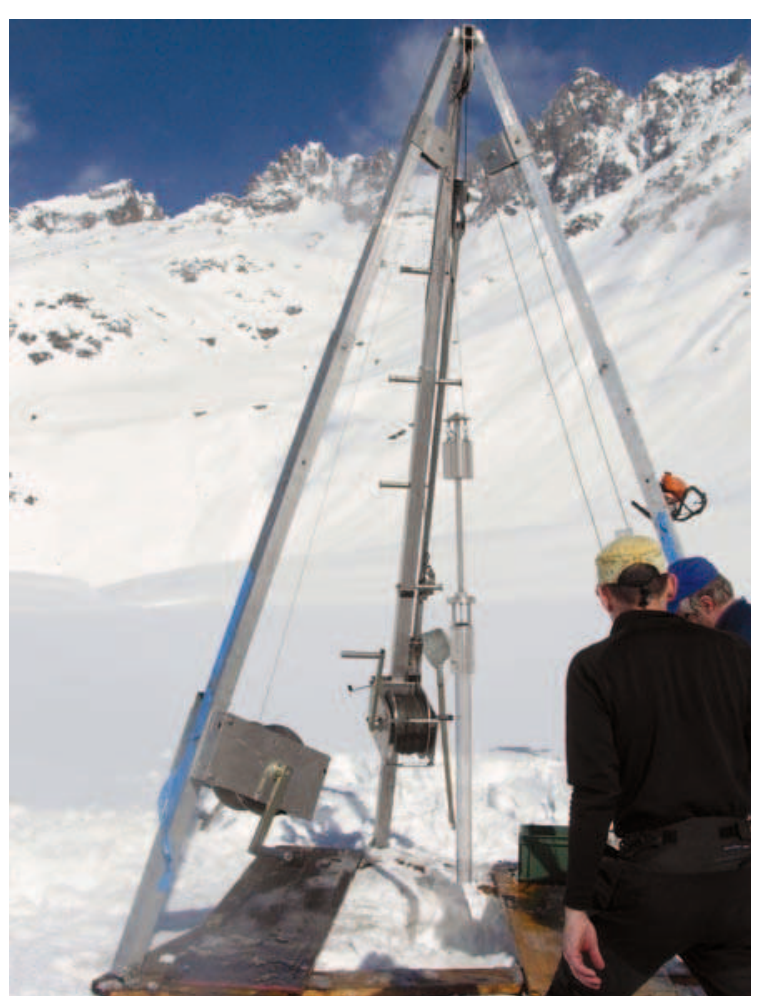

Abb. 2. Probennahme von Sedimentbohrkernen aus dem zugefrorenen Steinsee im Berner Oberland.

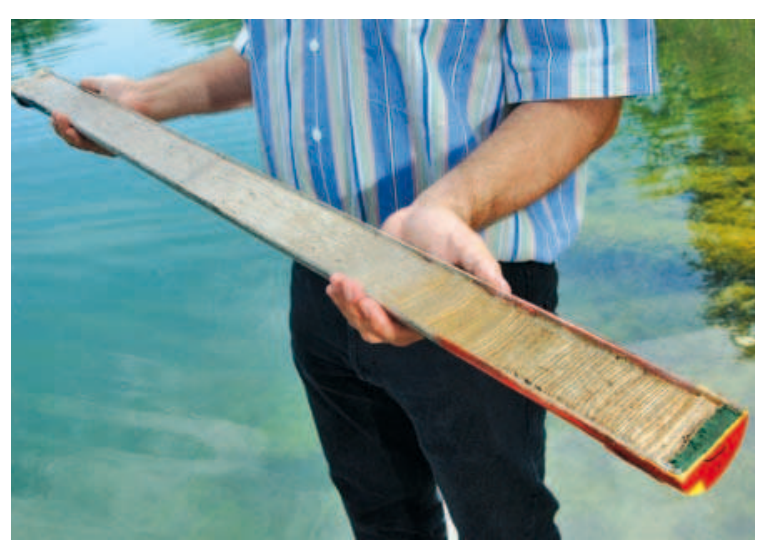

$A b b$. 3. Sedimentkern aus dem Greifensee bei Dübendorf in der Schweiz mit klar erkennbaren Jahreslagen: Die hellen Schichten sind Ausfällungen von Seekreide im Winter. (Foto: Ruedi Keller)

\section{QUERGELESEN}

\) Wissenschaftler untersuchen Alter und Schadstoffbelastung von See-Sedimenten. Dadurch können sie beurteilen, ob und wann Chemikalienverbote greifen.

》) Gletscher speichern persistente organische Schadstoffe (POPs). Die Chemikalien werden bei der Gletscherschmelze wieder frei und sammeln sich in den Sedimenten von Gletscherseen. In den letzten Jahren wurden aufgrund der Klimaerwärmung mehr POPs in den Sedimenten gefunden als noch vor zehn Jahren.

\> Neue Modelle können vorhersagen, wann Gletscher POPs wieder freisetzen. setzen sich mit ihnen im Sediment ab. Ist die Ablagerungsrate bekannt, lässt sich aus der Konzentration einer Substanz in der Sedimentschicht auf den flächenbezogenen atmosphärischen Eintrag schließen.

Vor 25 Jahren fand man beispielsweise heraus, dass die Dioxingehalte in See-Sedimenten aus schweizerischen Mittellandseen seit den 1940er Jahren stark ansteigen. Grund waren Emissionen aus der Abfallverbrennung und die umfassende Verwendung von chlororganischen Materialien wie Polyvinylchlorid (PVC). ${ }^{1)}$ Diesen Befund haben weitere Untersuchungen an anderen Gewässern bestätigt, und ähnliches war für andere POPs wie DDT und PCB zu beobachten., ${ }^{2,3}$

Wie neuere Messungen zeigen, nehmen die Schadstoffgehalte in den Sedimenten jetzt wieder ab. Die in den 1970er Jahren eingeleiteten Maßnahmen zur Reduktion der Emissionen sowie die eingeführten Anwendungsverbote waren demnach ein Erfolg. ${ }^{2,3,4)}$

\section{Sedimente als Geschichtsbücher}

- Um dem See Sedimente zu entnehmen, wird ein Kunststoffrohr mit einem Durchmesser von etwa sechs Zentimeter entweder im freien Fall oder durch Hammerschläge vertikal in den Seeuntergrund getrieben. Es nimmt so das Sediment auf (Abbildung 2). Solche Sedimentkerne können mehrere Meter lang sein. Im Labor werden sie längs aufgeschnitten und aufgeklappt, wobei der Schichtaufbau der Ablagerungen sichtbar wird. Wie die Seiten eines Geschichtsbuches zeugen die verschiedenen Sedimenttypen von vergangenen Umweltveränderungen.

Einige Seen besitzen ein Sedimentarchiv, in dem der saisonale Partikeleintrag in den See oder die Partikelproduktion im See an charakteristischen Jahreslagen (Warven) erkennbar ist (Abbildung 3). Diese Warven dokumentieren saisonale biochemische Prozesse wie Algenblüten oder Seekreideausfällungen, die durch Carbonatübersättigung des Wassers ausgelöst werden. Während man die Seekreide als dünne weiße Lage im Sediment erkennt, führen starke Algenblüten zur Ablagerung grünlicher Lagen, reich an Biomasse. Andererseits führen auch jährlich wiederkehrende anorganische Prozesse zu Warven: In proglazialen Seen unterscheidet sich beispielsweise die feinkörnige Winterlage klar von der grobkörnigen Sommerlage (Abbildung 4). Auch Extremereignisse wie Hochwasser sind in den Sedimenten erkennbar. Die Wassermassen im Hochwasser sind besonders reich an Schwebstoffen, die sich im tiefen Seebecken rasch ablagern.

\section{Das Alter eines Sediments bestimmen}

- Das Alter von Sedimentschichten lässt sich mit radioaktiven Isotopen abschätzen, die sich in den Sedimenten angesammelt haben. Beispielsweise gelangte das Isotop ${ }^{137}$ Cs bei Atombombentests in den 1960er Jahren sowie beim Reaktorunfall von Tschernobyl 1986 in die Umwelt. Es hat eine Halbwertszeit von 30,2 Jahren. Ein Sediment lässt sich auch über das Isotop ${ }^{210} \mathrm{~Pb}$ datieren (Halbwertszeit 22,3 Jahre), das kontinuierlich aus der Atmosphäre eingetragen wird.

Für weiter zurückliegende Perioden lässt sich mit der Radiokarbonmethode das Alter von abgelagertem organischem Material bestimmen, das in den See gespült wurde. ${ }^{14} \mathrm{C}$ hat eine Halbwertszeit von 5730 Jahren. Sind Warven vorhanden, so kann das Alter auch durch Abzählen ermittelt werden: Eine Warve entspricht einem Jahr.

Ist das Alter der Sedimentlagen bekannt, steht ein wertvolles Archiv zur Verfügung, mit dem sich Umwelt- und Klimaveränderungen der Vergangenheit rekonstruieren lassen. So dokumentieren Pollen im Sediment die Vegetationsentwicklung, Sauerstoffisotope in der Seekreide sind eine Funktion der Temperatur. Die Abfolge und Dicke charakteristischer Hochwasserablagerungen zeigen auf, wie oft Flutereignisse stattgefunden haben und wie intensiv diese waren.

POPs in See-Sedimenten werden mit der organischen Spurenanalytik 
Dichlordiphenyltrichlorethan (DDT)
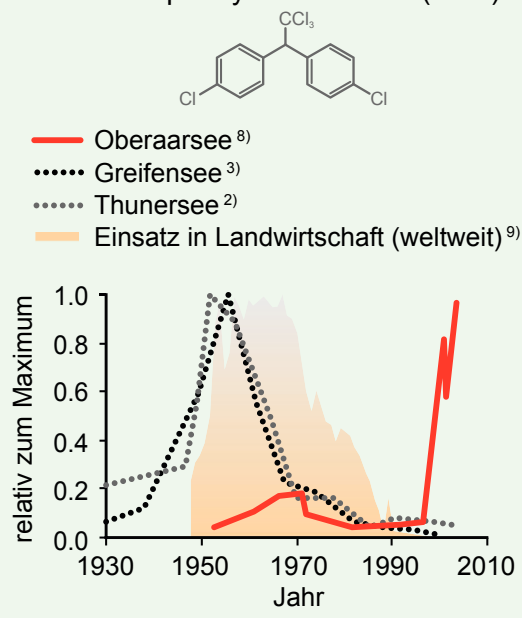

polychlorierte Biphenyle (PCB)

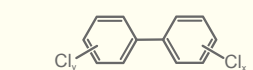

Oberaarsee ${ }^{8)}$

...... Greifensee ${ }^{3)}$

...... Thunersee ${ }^{2)}$

Produktion (weltweit) ${ }^{10}$

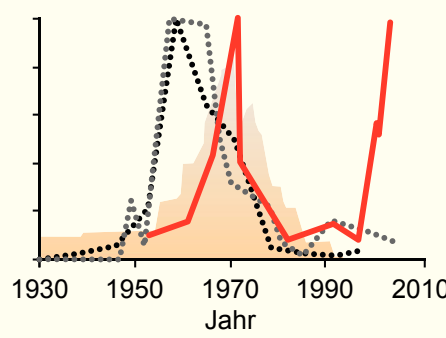

polychlorierte Dibenzo- $p$-dioxine und Dibenzofurane (PCDD/F)

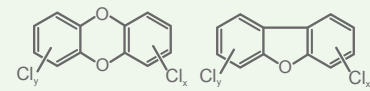

Oberaarsee ${ }^{8)}$

...... Greifensee ${ }^{3}$

...... Bodensee ${ }^{4)}$

Emissionen (Schweiz) ${ }^{11}$

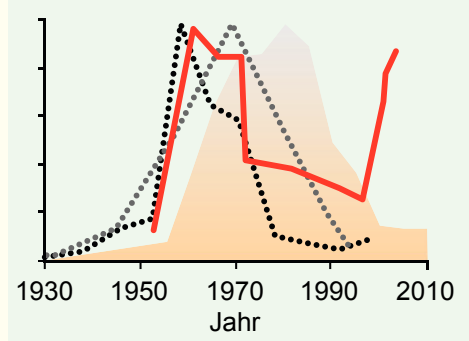

Abb. 5. Zeitlicher Verlauf des Eintrags von DDT und seinen Abbauprodukten, PCB sowie PCDD/F im Oberaarsee ${ }^{8)}$ und in schweizerischen Flachlandseen (Greifensee ${ }^{3)}$, Thunersee ${ }^{2)}$ und Bodensee ${ }^{4)}$ ). Zum Vergleich sind die eingesetzten, produzierten und emittierten Mengen von $D D T^{9)}, P C B^{10)}$ und $P C D D / F^{11)}$ dargestellt. Alle Darstellungen wurden auf den jeweiligen Maximalwert normiert.

men. ${ }^{6)}$ Ein solcher Trend konnte mit dem historischen Verlauf der Emissionen nicht erklärt werden. Die Beobachtung gilt daher als eine erste Bestätigung der Hypothese, dass durch die Klimaerwärmung die Gletscher schneller schmelzen und dadurch Schadstoffe freisetzen, die sie in früheren Jahren gespeichert haben. ${ }^{7)}$

\section{Gletscher als Speicher für Schadstoffe}

Zur weiteren Untermauerung dieses ersten Befunds untersuchten die Autoren einen Sedimentkern aus dem Oberaarsee, einem 2303 Meter hoch gelegenen, proglazialen Stausee im schweizerischen Berner Oberland (Abbildung 1). So konnte der zeitliche Verlauf des Eintrags verschiedener persistenter Chemikalien wie DDT, $\mathrm{PCB}$ und $\mathrm{PCDD} / \mathrm{F}$ rekonstruiert und mit früheren Untersuchungen in Flachlandseen verglichen werden (Abbildung 5). ${ }^{8)}$ Das Ergebnis: Für alle Chemikalien nimmt der Eintrag seit Ende der 1990er Jahre in Gletscherseen zu - in Flachlandseen hingegen bleiben die Konzentrationen weiterhin gleich niedrig oder nehmen sogar ab. Für einzelne Stoffe übertrifft der gegenwärtige Eintrag sogar den höchsten Eintrag zu Zeiten, als diese Stoffe noch breit verwendet und emittiert wurden. Da der Oberaarsee praktisch aus- schließlich vom zurzeit rasch abschmelzenden Oberaargletscher gespeist wird, müssen die Chemikalien aus dem Gletscher stammen. Dieser Speicher bildet damit eine sekundäre Quelle für die vor Jahrzehnten eingelagerten Stoffe.

Um den zeitlichen Verlauf der Freisetzung aus dem Gletscher auch quantifizieren und prognostizieren zu können, entwickelten die Autoren mit Unterstützung von Glaziologen ein Modell, das den Eintrag sowie das Fließ- und Schmelzverhalten des Gletschers berücksichtigt. Nach diesen Rechnungen werden die Stoffe, die in diesem Gletscher eingelagert sind, mit einer Zeitverzögerung von beinahe 50 Jahren wieder frei. Das klimabedingt verstärkte Abschmelzen beschleunigt diesen Vorgang. Bisher wurde demnach erst die Hälfte der ursprünglich gespeicherten Menge an POPs wieder freigesetzt.

Christian Bogdal ist seit 2009 als Umweltchemiker an der Eidgenössischen Technischen Hochschule (ETH) Zürich tätig. Er forscht auf dem Gebiet des Umweltverhaltens von persistenten organischen Schadstoffen und kombiniert dabei Spurenanalytik mit Massenbilanzmodellierung.

Peter Schmid ist seit 1996 analytischer Chemiker am schweizerischen Materialforschungsinstitut Empa (Swiss Federal Laboratories for Materials Testing and Research) und erforscht das Verhalten von anthropogenen persistenten Stoffen in der Umwelt.
Literatur

1) J. M. Czuczwa, F. Niessen, R. A. Hites, Chemosphere 1985, 14, 1175-1179.

2) C. Bogdal, P. Schmid, M. Kohler et al., Environ. Sci. Technol. 2008, 42, 6817-6822.

3) M. Zennegg, M. Kohler, P. C. Hartmann et al., Chemosphere 2007, 67, 1754-1761.

4) H. Hagenmaier, M. Walczok, Organohalogen Compd. 1996, 28, 101-104.

5) P. Schmid, M. Kohler, E. Gujer et al., Chemosphere 2007, 67, S16-S21.

6) C. Bogdal, P. Schmid, M. Zennegg et al., Organohalogen Compd. 2009, 71, 321-325.

7) J. M. Blais, D. W. Schindler, D. C. G. Muir et al., Ambio 2001, 30, 410-415.

8) C. Bogdal, P. Schmid, M. Zennegg et al., Environ. Sci. Technol. 2009, 43, 8173-8177.

9) Y. F. Li, R. W. Macdonald, Sci.Tot. Environ. 2005, 342, 87-106.

10) K. Breivik, A. Sweetman, J. M. Pacyna et al., Sci.Tot. Environ. 2002, 290, 181-198.

11) BAFU (Bundesamt für Umwelt), „Dioxine und Furane" (Hrsg: Buwal), Schriftenreihe Umwelt Nr. 290, Bern, 1997.

Flavio Anselmetti ist seit 2007 Geologe an der Eawag (Swiss Federal Institute of Aquatic Science and Technology). Er befasst sich mit Seeund Meeres-Sedimenten als Archive für Umwelt- und Klimaveränderungen, Naturgefahren und der Interaktion Mensch-Umwelt. Seit 2009 ist er Titularprofessor an der ETH Zürich. Martin Scheringer ist seit 1996 als Umweltchemiker an der Eidgenössischen Technischen Hochschule (ETH) Zürich tätig. Er beschäftigt sich mit der Verteilung anthropogener Substanzen in der Umwelt und der Risikobewertung von Chemikalien. 